\title{
DINÁMICA DE SISTEMAS Y CRECIMIENTO ECONÓMICO*
}

\author{
Josefa Ramoni Perazzi \\ Giampaolo Orlandoni Merli
}

" DOI: https://doi.org/10.18601/01245996.v24n46.07. Recepción: 03-032021, modificación final: 10-08-2021, aceptación: 29-11-2021. Sugerencia de citación: Ramoni P., J. y Orlandoni M., G. (2022). Dinámica de sistemas y crecimiento económico, Revista de Economía Institucional, 24(46), 115-132.

a $\mathrm{PhD}$ en Economía. Profesora titular, Escuela de Economía y Administración, Universidad Industrial de Santander, Bucaramanga, Colombia [jramonip@uis.edu.co].

b Doctor en Estadística. Profesor titular, Facultad de Ciencias Exactas, Naturales y Agropecuarias, Universidad de Santander, Bucaramanga, Colombia [gorlandoni@udes.edu.co]. 


\section{Dinámica de sistemas y crecimiento económico}

Resumen Este artículo muestra que la teoría económica se puede analizar mediante la dinámica de sistemas, un enfoque intuitivo que permite representar en forma gráfica los lazos de retroalimentación entre las variables que intervienen en los modelos. En particular, modela la visión del crecimiento de Adam Smith, según la interpretación muy simplificada de Robert Heilbroner, (desarrollada en foma más integral y exhaustiva por su maestro, el sociólogo y economista alemán Adolph Lowe) proponiendo un sistema computacional para el estudio de modelos complejos. El trabajo no pretende hacer un aporte original a la modelación económica sino mostrar una herramienta que permite hacer simulaciones con base en los principios de la dinámica de sistemas, utilizando software especializado; una herramienta que se puede utilizar en la enseñanza para mostrar opciones de modelación alternativas a las convencionales.

Palabras clave: modelos, crecimiento económico, dinámica de sistemas; JEL: O40, C60, C63

\section{System dynamics and economic growth}

Abstract This paper shows that economic theory can be analyzed by means of system dynamics, an intuitive approach that makes it possible to represent graphically the feedback loops between the variables involved in the models. It models Adam Smith's view of growth, according to Robert Heilbroner's highly simplified interpretation, (developed in a more comprehensive and exhaustive way by his teacher, the German sociologist and economist Adolph Lowe) by proposing a computational system for the study of complex models. The paper does not intend to make an original contribution to economic modeling, but to show a tool that allows simulations based on the principles of system dynamics, using specialized software. This tool can be used in teaching to show alternative modeling options to the conventional ones.

Keywords: Models, economic growth, systems dynamics; JEL: O40, C60, C63

\section{Dinâmica do sistema e crescimento econômico}

Resumo Este artigo mostra que a teoria econômica pode ser analisada por meio da dinâmica de sistemas, uma abordagem intuitiva que nos permite representar graficamente os loops de feedback entre as variáveis envolvidas nos modelos. Em particular, modela a visão de crescimento de Adam Smith, de acordo com a interpretação altamente simplificada de Robert Heilbroner, (desenvolvida de forma mais abrangente e exaustiva por seu professor, o sociólogo e economista alemão Adolph Lowe) propondo um sistema computacional para o estudo de modelos complexos. O trabalho não pretende dar uma contribuição original à modelagem econômica, mas mostrar uma ferramenta que permite simulações baseadas nos princípios da dinâmica de sistemas, utilizando softwares especializados; uma ferramenta que pode ser usada no ensino para mostrar opções de modelagem alternativas às convencionais.

Palavras-chave: modelos, crescimento econômico, dinâmica do sistema; JEL: O40, C60, C63 
T a complejidad creciente de los fenómenos socio económicos Lrequiere metodologías y herramientas para hacer análisis que ayuden a resolver problemas teóricos y prácticos. Los problemas del crecimiento económico han sido abordados desde distintos enfoques y concepciones teóricas. La escuela clásica creó un cuerpo teórico coherente iniciado por Adam Smith, uno de sus principales exponentes. $\mathrm{El}$ análisis tradicional consiste aislar los elementos fundamentales y establecer entre ellos relaciones lógicas y formales que se representan en modelos que buscan explicar y predecir su comportamiento. Esos elementos son parte de fenómenos complejos e interrelacionados por vínculos de retroalimentación, cuyo análisis y solución requieren métodos y herramientas innovadoras que asuman esa complejidad. La dinámica de sistemas es un enfoque que permite analizar en forma gráfica el objeto de estudio, utilizando un lenguaje de íconos que representan los elementos o variables del sistema, no solo siguiendo una lógica sino también una secuencia. Así, sus relaciones teóricas y matemáticas se traducen en modelos gráficos, basados en ecuaciones diferenciales que permiten simular la dinámica del sistema en cuestión. Además, se pueden introducir cambios que producen distintas trayectorias o comportamientos, por ejemplo, cambios en las variables de estado iniciales, y hacer análisis de sensibilidad al cambio de los parámetros (Radzicki y Sterman, 1994).

Este artículo analiza la teoría del crecimiento económico de Adam Smith, según la simplificada interpretación de Robert Heilbroner, con el enfoque de la dinámica de sistemas (DS); construye algunos modelos, que, usando las técnicas apropiadas, permiten hacer simulaciones y generar trayectorias dinámicas de las variables de estado incluidas en esos modelos.

Los sistemas económicos se pueden modelar mediante ecuaciones diferenciales, lineales y no lineales, y su comportamiento se estudia utilizando los métodos clásicos de resolución analítica, aunque para estructuras más complejas hay que recurrir a métodos numéricos apropiados. La DS modela estos sistemas utilizando un lenguaje gráfico de programación, donde los íconos representan variables de estado y variables de flujo. Los modelos gráficos se crean utilizando cuatro íconos: niveles o acumulados (stocks), flujos (flows), convertidores y conectores. Las ecuaciones y valores de los parámetros se especifican para los niveles, los flujos y los convertidores. A partir del modelo gráfico, el software genera las ecuaciones que lo describen, las cuales se resuelven por métodos clásicos de análisis numérico (Euler, 
Runge-Kutta), ajustando el intervalo de tiempo para el cálculo, y el tiempo de ejecución para la simulación. Se obtienen entonces datos, en forma tabular, y gráficas de las trayectorias de las variables, como series temporales o diagramas de fases. Además de resolverlas, los sistemas modelados se pueden simular variando los parámetros de sus ecuaciones, obteniendo las trayectorias dinámicas de las variables de estado involucradas. Así se formulan, analizan y simulan modelos que generan comportamientos dinámicos de las variables económicas, interpretando teorías clásicas de crecimiento económico (Orlandoni y Ramoni, 2018a, 2018b).

En 1968, bajo la dirección de Aurelio Peccei, en la Accademia Nazionale dei Lincei, Roma, se reunieran algunos investigadores para tratar problemas complejos como la pobreza creciente, la degradación del medio ambiente, la urbanización descontrolada, la inseguridad laboral, la inflación y los desajustes económicos. Así se inició el Club de Roma y su proyecto sobre el futuro de la humanidad, que buscaba crear conciencia sobre estos problemas, los cuales tienen tres características comunes: afectan a todas las sociedades; intervienen aspectos sociales, económicos y políticos; y existe una un alto grado de interacción entre ellos. Los investigadores plantearon que había un reconocimiento general de esos problemas pero que no se entendían muy bien sus orígenes ni las interrelaciones entre sus diversos componentes, de modo que no se podían proponer soluciones efectivas. En gran parte, porque no se adoptaba un enfoque sistémico, y se seguían analizando sus elementos sin comprender que el todo es más que la suma de sus partes, es decir, sin entender que el mundo es un sistema de componentes en interacción.

El profesor Jay Forrester, del Instituto de Tecnología de Massachusetts, presentó un modelo que explicaba la problemática mencionada de una manera global y propuso utilizar la dinámica de sistemas para analizar el comportamiento y las relaciones de esos componentes (Meadows et al., 1972).

Jay Forrester (1918-2016) fue entonces el pionero de la dinámica de sistemas. Con base en la teoría de servomecanismos y control, incorporó el concepto de retroalimentación (feedback) y de autorregulación sistémica. Publicó varios libros en los que fundamentó la teoría aplicó las técnicas de la DS al análisis de los problemas mencionados por el Club de Roma. En sus libros trata problemas de la dinámica industrial, la dinámica urbana y la dinámica mundial. 


\section{MÉTODOS}

La economía es una disciplina social de carácter lógico-empírico que describe, explica y predice fenómenos socioeconómicos, proporcionando a través de teorías económicas relaciones de causalidad entre variables relevantes, base para la construcción de modelos económicos. Los sistemas socioeconómicos, por su continuo intercambio de entradas y salidas con el medio ambiente, son sistemas no lineales, abiertos, disipativos e incorporan mecanismos de descarga que amortiguan sus respuestas ante los flujos de entrada; además incorporan estructuras de retroalimentación negativa que compensan los efectos de las entradas para mantener el estado del sistema en sus niveles deseados (Radzicki y Sterman, 1994).

En este trabajo, basándose en la metodología de DS, se estudia la estructura de retroalimentación de sistemas complejos que genera su comportamiento dinámico; se identifican y describen las fuerzas que surgen internamente en esos sistemas y que producen los cambios observados a lo largo del tiempo, explicando cómo se relacionan dichas fuerzas (Forrester, 1961; Nijkamp y Reggiani, 1992). Estos procedimientos se operacionalizan mediante software apropiado, como Powersim, Vensim, Stella, entre otros, que permite modelar, resolver y simular el sistema en estudio; en este trabajo se utiliza Powersim Studio 10 Express (Powersim, 2017).

\section{MODELOS ECONÓMICOS Y DINÁMICA DE SISTEMAS}

En general, los sistemas dinámicos se caracterizan por tener estructuras internas de retroalimentación que generan su comportamiento dinámico; la DS permite representar gráficamente esas estructuras complejas, describiendo los mecanismos internos que producen cambios en el tiempo, además de analizar la manera cómo se interrelacionan (Forrester, 1961; Richardson, 1995). Los modelos de DS se construyen para explicar y predecir efectos de decisiones alternativas; para ello se identifican los elementos básicos del sistema en estudio y las estructuras de retroalimentación que explican su comportamiento dinámico, se construyen modelos lógico-matemáticos del comportamiento del sistema para simular sus trayectorias bajo diferentes escenarios, y se enfatiza el análisis de las tasas de cambio de las diferentes magnitudes que definen el sistema estudiado (Ruth,2012). 


\section{MODELACIÓN MEDIANTE DINÁMICA DE SISTEMAS}

Según el enfoque de DS, el proceso de modelado se centra en la visión global del problema, iniciando desde la conceptualización y finalizando en la formulación de ecuaciones y pruebas del modelo. La visión global de la metodología se basa en dos supuestos interrelacionados: la búsqueda de objetivos y la estructura de retroalimentación del sistema. La búsqueda de objetivos requiere la presencia de ciertas estructuras internas de retroalimentación negativa, definidas como procesos circulares en los que cualquier desviación de las condiciones actuales con respecto a las condiciones deseadas induce acciones para que el sistema regrese al estado deseado; así, el comportamiento que desarrolla el sistema no solo es consecuencia de impactos externos, sino de la manera como la estructura de retroalimentación del sistema procesa dichos impactos (Forrester, 1961; Radzicki y Sterman, 1994; Richardson, 1995; Richmond, 1985).

$\mathrm{El}$ enfoque de DS se puede resumir del modo siguiente: determinar los limites apropiados para decidir qué elementos incluir en el sistema, pensar en relaciones causa-efecto, enfocarse en las relaciones de retroalimentación entre los componentes, considerando la estructura del sistema como una red causal de lazos de retroalimentación que explica el comportamiento dinámico de los elementos del sistema a lo largo del tiempo.

\section{LAZOS DE RETROALIMENTACIÓN EN DINÁMICA DE SISTEMAS}

Se identifican dos tipos de lazos de retroalimentación en DS, retroalimentación positiva $(\mathrm{RP})$ y retroalimentación negativa $(\mathrm{RN})$. La $\mathrm{RP}$ produce un efecto exponencial de crecimiento: si una magnitud comienza a crecer, dicho crecimiento no se detiene en el tiempo, sino que se mantiene a esa tasa; por ejemplo, si aumenta la población total, entonces aumenta el número de nacimientos, produciendo un aumento proporcional de la población y, a su vez, otro aumento sucesivo en el número de nacimientos. Por otra parte, la RN tiende a mantener el sistema bajo control, dentro de un cierto rango de variación limitado, causando comportamientos estables por el principio de autorregulación; así, el aumento en la población genera incrementos en la mortalidad, lo que conduce a una disminución en su nivel y, con cierto retardo, produce una disminución en la mortalidad. (Forrester, 1961). 


\section{COMPONENTES DE UN MODELO DE DINÁMICA DE SISTEMAS}

Los modelos de DS se estructuran mediante variables y ecuaciones de flujo y de estado o nivel; brevemente se describen a continuación:

Variables de estado (variables tipo stock): representan el estado del sistema en un momento dado, causado por la acumulación de recursos a lo largo del tiempo, resultado de la diferencia entre los flujos de entrada y los flujos de salida para determinado nivel.

Variables de flujo (variables tipo flow): definen el flujo de material o energía entre variables de estado, determinando los cambios en dichas variables por unidad de tiempo.

Ecuaciones de nivel: representan el nivel acumulado de una variable, y relacionan su magnitud en $t$ con su valor en $t-1$ y con su tasa de cambio, en el intervalo de cálculo $d t$. La estructura general es $N_{t}=N_{t-1}$ $+d t\left(\operatorname{Tasa}_{(t-1 ; t)}\right)$. En el caso de un crecimiento demográfico, la ecuación de nivel se expresa como $P_{t}=P_{t-1}+d t(N a t-\text { Mort })_{(t-1 ; t)}$.

Ecuaciones de flujo: representan tasas de variación de las variables de nivel en cada unidad de tiempo y definen el comportamiento del sistema.

\section{MODELOS Y EJERCICIOS: EL CRECIMIENTO EN ADAM SMITH}

Siguiendo la interpretación de Heilbroner acerca de la teoría del crecimiento económico de Adam Smith, expuesta en La riqueza de las naciones, donde las leyes del mercado se sintetizan en el concepto de la mano invisible, la atención se centra en la dinámica de la economía capitalista, su productividad y su evolución a largo plazo. Adam Smith se pregunta qué es lo que impulsa a la sociedad a multiplicar la riqueza. Según Smith, es en parte, el mecanismo del mercado y su incansable actividad, al que se añaden dos elementos que rigen su comportamiento y lo impulsan en una espiral ascendente de productividad. El primero es la ley de la acumulación y el segundo es la gran ley del sistema: la ley de la población (Heilbroner, 1998).

\section{Ley de la acumulación}

La actividad más importante del capitalista es la acumulación de los beneficios, que permite establecer nuevas y mejores fábricas, con mejor maquinaria que incrementa la productividad del trabajo, lo que induce un aumento aún mayor del producto y del consumo, y así mejora el bienestar de toda la sociedad (capitalistas y trabajadores). A medida que los beneficios de los capitalistas se reinvierten en fábricas y máquinas más avanzadas tecnológicamente, y como la oferta de trabajo 
es finita, la competencia por la fuerza laboral presiona a los salarios al alza, lo que frena el crecimiento de los beneficios, y con ello detiene la espiral de acumulación y por ende el crecimiento económico.

El diagrama de la ley de la acumulación muestra este comportamiento y sus dos estructuras de retroalimentación: el lazo positivo superior, que representa la acumulación de beneficios, y el lazo inferior negativo, mediante el cual la acumulación de beneficios frena la mayor acumulación. La acumulación de maquinaria implica mayor demanda de trabajadores, lo que eventualmente conduciría a salarios más altos hasta que las ganancias, principal incentivo de la acumulación, se reducen (ver gráfica 1, ley de la acumulación).

Gráfica 1

Mapa conceptual. Ley de la población y ley de la acumulación

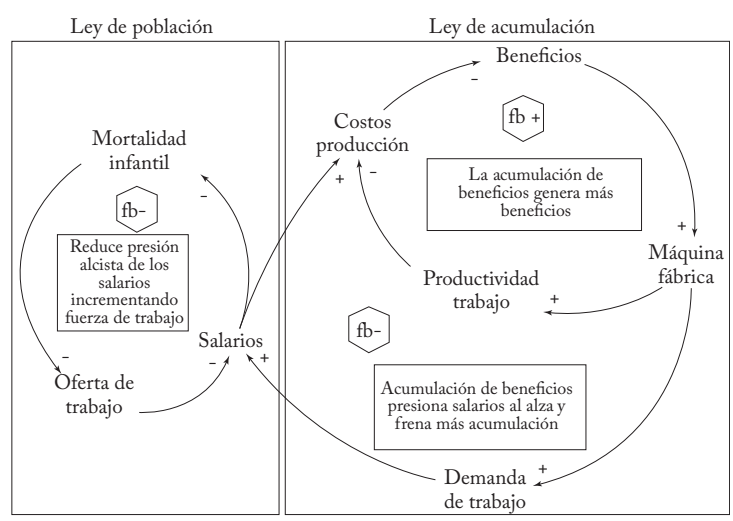

Fuente: Heilbroner (1998), elaboración propia.

\section{Ley de la población}

La ley de la población ayudaría a superar dicho obstáculo para el crecimiento. Las fuerzas del mercado laboral regulan la cantidad de trabajo disponible por la interacción entre oferta y demanda: mayores salarios crean condiciones para más producción y bienestar, y se ofrece más trabajo, debido a la reducción de la mortalidad infantil.

Cuando Smith escribe "así es como la demanda de hombres, como la de cualquier otra mercancía, necesariamente regula la producción de hombres" (Smith, 1978, p. 98), se está refiriendo, más que a nuevos nacimientos, a reducción en mortalidad infantil, debido a mejoras en las condiciones de vida de la población trabajadora.

Cuando aumenta la fuerza laboral se reduce la presión alcista de los salarios; la retroalimentación positiva actúa con cierto retardo y 
fortalece así la espiral de acumulación. E1 retardo en la creación de fuerza laboral, luego de reducir la mortalidad infantil, actúa en contra, generando recesiones periódicas en la economía (Richmond, 1985), (ver gráfica 1, ley de la población).

En suma, tres lazos de retroalimentación explican la dinámica del crecimiento económico. La ley de la producción aporta dos lazos: un lazo de RP, por el que la acumulación de beneficios genera mayor acumulación; el otro es un lazo de $\mathrm{RN}$, pues la misma acumulación crea condiciones que frenan la mayor acumulación, al reducir la oferta de trabajo. La ley de la población proporciona a su vez, un lazo RN: a medida que el aumento de salarios reduce los beneficios, desacelerando el proceso de acumulación, al mismo tiempo está actuando a favor del aumento en la fuerza laboral, por cuya escasa oferta se produjo un incremento en salarios. Entonces, este lazo RN opera contrarrestando al anterior lazo RN de la ley de producción; ambos lazos regulan la oferta laboral y determinan el nivel salarial.

Este juego entre los dos lazos RN activa el lazo RP, y esta mecánica explica las recesiones periódicas observadas en el proceso de expansión económica. Esta estructura refleja la visión optimista que AS tiene del sistema capitalista: un futuro de constante expansión, acompañado de periódicas recesiones, pero proporcionando mejores condiciones de vida, tanto para trabajadores como para empresarios capitalistas.

\section{LA DINÁMICA DEL SISTEMA DE CRECIMIENTO CLÁSICO}

Para traducir estas ideas a un mapa de DS, hay que diagramar los lazos de retroalimentación. Conviene partir del estado estacionario para facilitar la interpretación y análisis de las simulaciones.

En una estructura que representa el proceso de retroalimentación positiva, el beneficio del sistema acumula la diferencia entre ingresos generados por la compra de bienes y los gastos causados por el pago de salarios y reinversiones de los beneficios en nuevas y mejores maquinarias, acumuladas en un stock de máquinas que incrementan la productividad del trabajo y permiten el aumento en la demanda de trabajadores. El resultado es un incremento de producción, acumulado en el inventario de bienes, el cual se va reduciendo por el aumento de la demanda y posterior venta. Los salarios se regulan por la relación entre oferta y demanda de trabajo, que se supone disponible sin restricciones; además, los precios de los bienes se suponen constantes, pero este supuesto se relaja en análisis posteriores.

En estado estacionario, los ingresos se distribuyen en pagos de salarios y en inversiones, por lo que los beneficios no crecen; el stock de 
maquinaria es constante (la inversión en nuevas máquinas se produce a una tasa equivalente al retiro de maquinaria depreciada y obsoleta); significa que no hay avance tecnológico y la productividad del trabajo es constante, lo que conduce a un stock constante de trabajo igual al trabajo deseado. La producción es constante e igual a la demanda; los inventarios no varían y la economía está en un estado de equilibrio perfectamente balanceado.

\section{EJERCICIOS DE SIMULACIÓN CON EL MODELO DE CRECIMIENTO DE ADAM SMITH}

La gráfica 1 anterior muestra el mapa conceptual de relaciones causales, junto con los lazos de retroalimentación identificados, que definen el sistema de crecimiento económico de Adam Smith. E1 sistema queda conformado por los dos lazos de RN y un lazo RP, ya comentados.

Gráfica 2

Ejercicio 1 Estructura del crecimiento económico. Estado estacionario

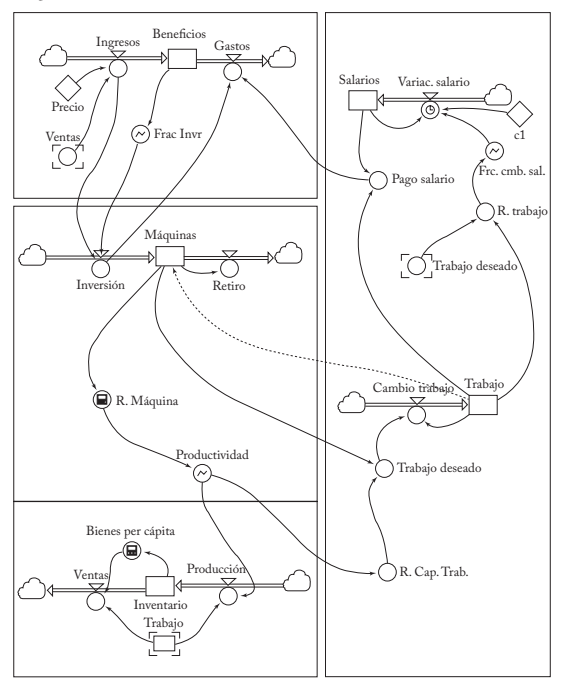

Fuente: Heilbroner (1988) y Richmond (1985); elaboración propia con Powersim Studio.

La gráfica 2 representa el modelo de crecimiento económico en estado estacionario, en lenguaje de DS, y muestra las relaciones causales $\mathrm{y}$ las trayectorias de las principales variables. E1 modelo contiene relaciones matemáticas, ecuaciones y valores de los parámetros que definen el sistema; esas relaciones se describen en el anexo (cuadro A1). Partiendo del modelo básico en estado estacionario, se pueden 
hacer diversos ejercicios, como variar el valor de parámetros, o modificar relaciones funcionales entre sus variables, y así observar el funcionamiento del modelo diseñado y la respuesta de las principales variables que lo definen. El cuadro 1 resume las características de los ejercicios realizados.

Cuadro 1

Ejercicios realizados con el modelo económico de crecimiento

\begin{tabular}{ll}
\hline Ejercicio & Tipo de intervención \\
\hline Ejercicio 1 & Estado estacionario de la economía \\
Ejercicio 2 & Restricción de la fuerza laboral \\
Ejercicio 3 & Reducción de mortalidad infantil y aumento de fuerza laboral \\
Ejercicio 4 & Espiral precio-salario. Precios función de salarios \\
\hline
\end{tabular}

Fuente: elaboración propia.

\section{Ejercicio 2: Introducción de restricciones en la fuerza laboral}

Esta restricción, expresada como un volumen finito de oferta de trabajo, se modela mediante un stock de fuerza laboral, que ofrece y recibe trabajo. El propósito es ver el efecto que, sobre la estructura previa, tiene la imposición de esa restricción laboral. La gráfica 3 muestra la modificación hecha al modelo inicial en equilibrio, incorporando la restricción indicada.

Gráfica 3

Ejercicio 2: Incorporación de restricción en la fuerza laboral

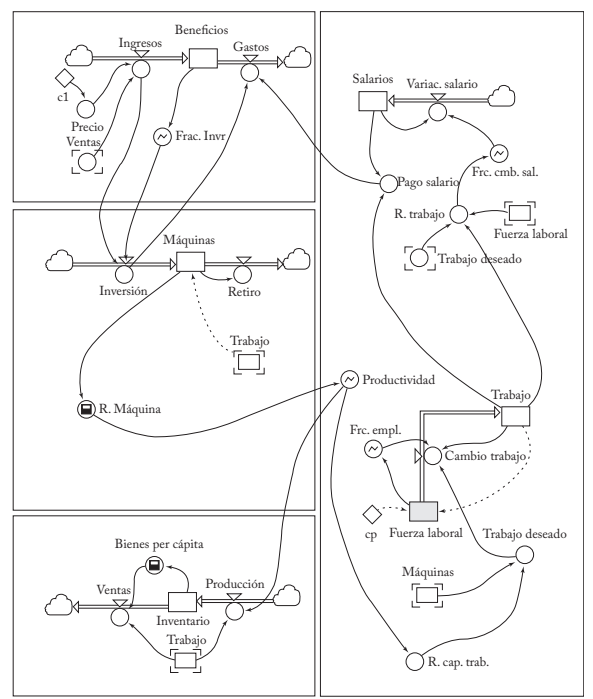

Fuente: elaboración propia con Powersim Studio. 
La modificación del modelo inicial consiste en añadir un depósito fijo (Fuerza Laboral) del cual se extrae y al que llega trabajo. El efecto de imponer esta restricción en la oferta laboral es frenar el crecimiento económico, debido a la restricción que a su vez se impone a la espiral de acumulación de beneficios. En la gráfica 4 se muestra la consecuencia de esta restricción sobre los salarios, el trabajo y la productividad. La expansión económica previamente observada se frena, al chocar con esta restricción en la fuerza laboral, corroborando la explicación intuitiva de Smith.

Gráfica 4

Ejercicio 2: Restricción laboral
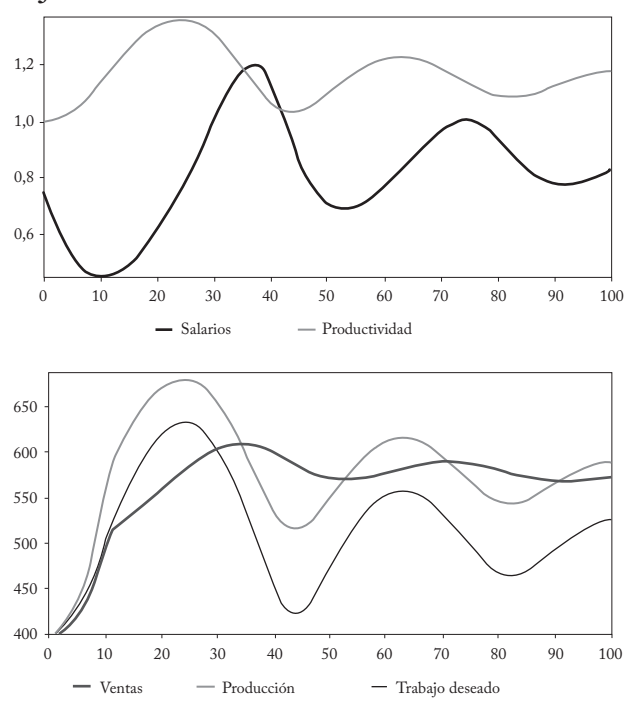

Fuente: elaboración propia con Powersim Studio.

La simulación muestra el surgimiento de un ciclo estacionario, en el sentido de que la economía, aún frenada en su capacidad de crecer por la restricción de la fuerza laboral, trata de despegar. Este movimiento cíclico estacionario, de alguna manera está alimentado por fluctuaciones salariales, que a su vez reflejan las oscilaciones de los beneficios, reinvertidos en capital. Como la fuerza laboral está restringida a un nivel de pleno empleo, aun cuando el trabajo deseado sea menor que el nivel de trabajo real, éste no se ajusta a niveles inferiores, ya que la variable "cambio en trabajo" se anula en el momento en que se alcanza el pleno empleo. 


\section{Ejercicio 3: Reducción en la mortalidad infantil}

Cuando la oferta de fuerza laboral es escasa, los salarios aumentan, lo que mejora el estándar de vida de los trabajadores y reduce la mortalidad infantil, de modo que la oferta laboral aumenta, aunque con cierto retardo. Al salir del estado de equilibrio, la economía entra en un crecimiento cíclico, y queda sujeta a recesiones periódicas, debido a elevados niveles de salarios, resultado de la escasa oferta laboral; ello causa reducción de los beneficios y caída de las inversiones en capital, con la consecuente reducción en el crecimiento de la productividad, desembocando en recesión. La consecuencia es un ajuste a la baja en los salarios, como respuesta al incremento en la fuerza laboral, resultado de la reducción en la mortalidad infantil que previamente se produjo por los altos salarios que se alcanzaron.

Para implementar esta reducción en mortalidad infantil, se incorpora al modelo una variable de flujo (IncrLabor) que afecta a la FuerzaLaboral, antes definida; el volumen de ese incremento de labor depende del nivel de los salarios de manera directa; al reducirse la mortalidad infantil, por efecto de niveles más altos de salarios, mayor va a ser dicho incremento. La gráfica 5 muestra la evolución de las principales variables en este ejercicio.

Gráfica 5

Ejercicio 3: Reducción de la mortalidad infantil
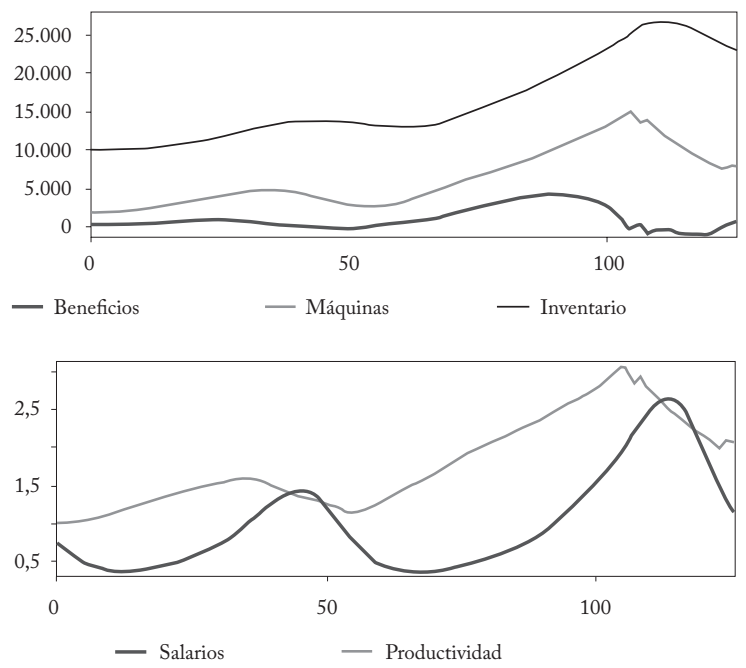

Fuente: elaboración propia con Powersim Studio. 
La estructura que incorpora al modelo la reducción en la mortalidad infantil se muestra en la gráfica 6, resaltando en gris las modificaciones necesarias para su implementación.

Gráfica 6

Ejercicio 3: Reducción de mortalidad infantil y aumento de fuerza laboral

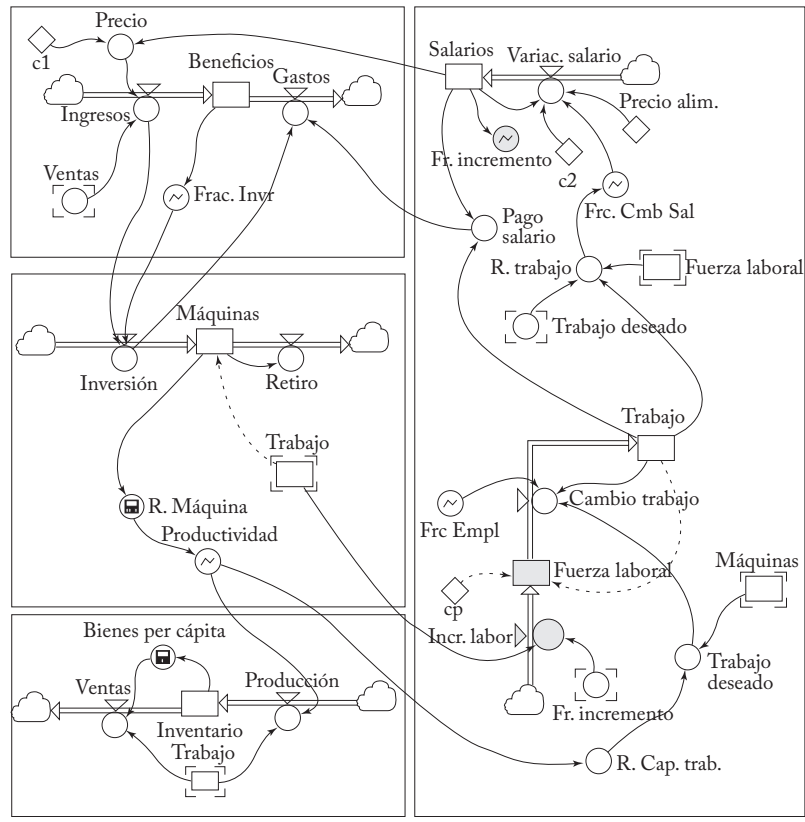

Fuente: elaboración propia con Powersim Studio.

\section{Ejercicio 4: Espiral precio-salario. Precio en función de salario}

El diagrama anterior, que se muestra en la gráfica 6, ya incorpora la relación entre precio y salario (aunque allí el precio sigue constante, al inactivar esa relación mediante $\mathrm{c} 1=0$ ).

La relación precio- salario permite que los capitalistas trasladen sus costos de producción en forma de precios crecientes para los bienes de consumo que ellos producen.

La gráfica 7 muestra los resultados de esta nueva estructura; allí se observa la espiral de crecimiento de la economía, a través de la dinámica de las variables que intervienen: productividad, salarios, trabajo y beneficios crecientes. Además, con esta modificación surge una nueva espiral en la economía: la espiral precio-salario. 


\section{Gráfica 7}

Ejercicio 4: Precios variables. Espiral precio-salario

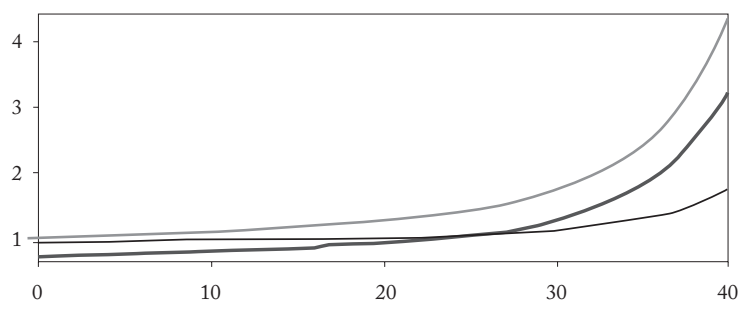

- Salarios - Precio - Productividad

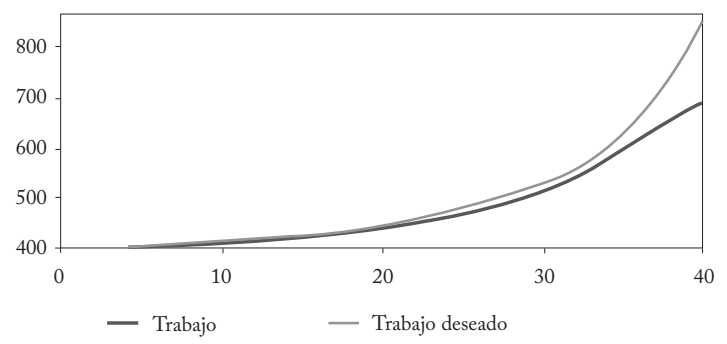

Fuente: elaboración propia con Powersim Studio.

\section{CONCLUSIONES}

Los modelos son estructuras teóricas simplificadas de la realidad que permiten entender el funcionamiento de los sistemas que representan, así como manipular, simular y optimizar las variables de interés con el fin de explicar su estructura interna y su comportamiento dinámico.

Para entender mejor las complejas interrelaciones de los fenómenos socioeconómicos es imprescindible la modelación dinámica de los sistemas en los que se inscriben. Esta tarea ha sido facilitada por la disponibilidad de potentes computadores personales, así como de software amigable que permite hacer programación gráfica, como Vensim, Powersim, Stella, entre los más conocidos. Así se crean espacios de aprendizaje y análisis de sistemas dinámicos, en los que se pueden simular trayectorias que representan posibles dinámicas futuras. Aquí es importante la abstracción de los hechos reales al hecho modelado, el paso más complejo de la modelación, pues centra la atención en las características más relevantes de las estructuras que generan las dinámicas observadas en los sistemas de interés.

La DS, como método de análisis sistémico, es una poderosa herramienta para modelar la visión global de los fenómenos en diversos campos: biología, política, sociología, economía, ecología, salud. 
Algunos ejemplos de modelos relevantes son los modelos mundiales del Club de Roma (World1, World2, World3), los modelos de epidemias SIR, muy utilizados en esta época de Covid-19, los modelos de comportamiento organizacional, los de cadenas de suministros, de manejo de recursos naturales, etc. (Gilbert y Troitzsch, 2005).

En economía, la modelación de las estructuras económicas mediante DS permite comprender, explicar y simular la trayectoria de las principales variables, así como probar diferentes políticas económicas en escenarios específicos. En este trabajo se presenta un esquema simplificado la teoría clásica de crecimiento económico de Adam Smith, sintetizado por Heilbroner y visto bajo el lente de la DS. Luego de formular y simular el modelo básico de crecimiento, se hacen diversos ejercicios para probar hipótesis relevantes.

Partiendo de una economía en estado estacionario, como ejercicio 1 , se hacen varias intervenciones. El ejercicio 2 muestra cómo se puede frenar el crecimiento económico con restricciones en la fuerza laboral, comparando con el caso de fuerza laboral siempre disponible.

La economía tiene mecanismos que pueden causar movimientos cíclicos sin necesidad de que crezca la oferta laboral, lo que, según Adam Smith, era necesario para generar dichos ciclos. El espíritu de libre mercado con el cual Smith concibe la economía capitalista plantea que el sistema tiene su propio remedio si se deja actuar libremente. Cuando la oferta laboral es fija o limitada y hay competencia entre los capitalistas por aumentar la producción y por tanto demandan más trabajo, los salarios se incrementan, lo que mejora el bienestar de los trabajadores; para Smith, esto es fundamental para reducir la mortalidad infantil, que fortalece y aumenta la oferta laboral a largo plazo. En el ejercicio 3 se prueba la hipótesis de que, al disminuir la mortalidad infantil por una mejora de los salarios, a largo plazo se produce un crecimiento sostenible con recesiones periódicas. Finalmente, el ejercicio 4 interviene los precios hasta ahora fijos, y define precios variables, dependientes de los salarios, generándose así, además de la espiral de crecimiento, una nueva espiral, la espiral precio-salario.

En suma, la DS puede representar y modelar teorías relativamente complejas, debido a las estructuras de retroalimentación inherentes a esas teorías; una de las fortalezas del enfoque de DS es el poder identificar y plasmar esos lazos de retroalimentación. Ello posibilita analizar de manera más intuitiva y dinámica las relaciones de causaefecto contenidas en los modelos matemáticos, sabiendo que los métodos numéricos requeridos para resolver y simular dichos modelos, generalmente expresados como sistemas de ecuaciones diferenciales 
no lineales, son confiables y están incorporados en software apropiado, tal como Powersim, usado en este caso.

Cabe resaltar que el artículo no pretende hacer un aporte original a la modelación económica sino mostrar una herramienta de modelación que, pese a sus desarrollos realistas, ha sido relegada por la modelación abstracta y formalista, y que no debería ser ignorada en la enseñanza de la economía. La continuación de este ejercicio conceptual es dar realismo a estos esquemas para aplicarlos al estudio de economías concretas. Para ello hay que calibrar los modelos, identificar las relaciones sus variables estructurales y obtener valores reales de los parámetros. Esa tarea será objeto de un trabajo posterior.

\section{ANEXO}

Cuadro A1

Ecuaciones del modelo de crecimiento económico

\begin{tabular}{|c|c|c|c|}
\hline \multicolumn{2}{|l|}{ Name } & Definition & \multirow{2}{*}{$\begin{array}{l}\text { Variable Type } \\
\text { level }\end{array}$} \\
\hline 甲… & INVENTARIO & 10000 & \\
\hline 由 & TRABAJO & 400 & \multirow{2}{*}{$\begin{array}{l}\text { level } \\
\text { level }\end{array}$} \\
\hline 吕 & BENEFICIOS & 40 & \\
\hline$\oplus$ & Fuerzalaboral & CP-TRABAJO & \multirow{2}{*}{ level } \\
\hline 田品 & MAQUINAS & 4"TRABAJO & \\
\hline 由 & SALARIOS & 0,75 & $\begin{array}{l}\text { level } \\
\text { level }\end{array}$ \\
\hline & c1 & 1 & constant \\
\hline & $c_{2}$ & 1 & constant \\
\hline 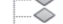 & сp & 0,25 & constant \\
\hline 8 & PrecioAlim & 0,1 & constant \\
\hline 0 & Pago_Salario & TRABAJO*SALARIOS & auxiliary \\
\hline$=\infty$ & PRODUCCION & TRABAJO*PRODUCTIVIDAD & auxiliary \\
\hline$=\infty$ & VENTAS & TRABAJO=Bienes_per_Capita & auxiliary \\
\hline$=\infty$ & Variac_Salario & SALARIOS*(Frc_Cmb_Sal+c2"PrecioAlim) & auxiliary \\
\hline$=0$ & INGRESOS & Precio*VENTAS & auxiliary \\
\hline 0 & Trabajo_Deseado & MAQUINAS/R_Cap_Trab & auxiliary \\
\hline 8 & R_Maquina & MAQUINAS/INIT(MAQUINAS) & auxiliary \\
\hline$=\infty$ & RETIRO & MAQUINAS/16 & auxiliary \\
\hline & GASTOS & INVERSION+Pago_Salario & auxiliary \\
\hline$=\infty$ & INVERSION & INGRESOS*Frac_Invr & auxiliary \\
\hline 8 & Fr_lncremento & GRAPH(SALARIOS;0;0,75; $\{0 ; 0,0065 ; 0,0125 ; 0,0168 ; 0,0215 ; 0,0255 ; 0,0283 ; 0,0305 ; 0,032 ; 0,033 ; 0,0333 / /$ Min:0;Max:0.04//1) & auxiliary \\
\hline 8 & Frc_Cmb_Sal & GRAPH(R_Trabajo; $0 ; 0,2 ;\{0,25 ; 0,18 ; 0,133 ; 0,09 ; 0,05 ; 0 ;-0,0675 ;-0,12 ;-0,172 ;-0,212 ;-0,25 / /$ Min:-0.25;Max:0.25//3) & auxiliary \\
\hline & PRODUCTIVIDAD & GRAPH(R_Maquina; $0 ; 1 ;\{0,64 ; 1 ; 1,32 ; 1,56 ; 1,84 ; 2,1 ; 2,32 ; 2,54 ; 2,74 ; 2,98 ; 3,2 / /$ Min: $0.5 ;$ Max:4///\}) & 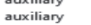 \\
\hline & Frc_Empl & GRAPH(FuerzaLaboral; $0 ; 10 ;\{0 ; 0,185 ; 0,345 ; 0,51 ; 0,66 ; 0,755 ; 0,835 ; 0,905 ; 0,96 ; 0,98 ; 0,995 / /$ Min:0;Max: $100 / / 1)$ & auxiliary \\
\hline & Frac_Invr & GRAPH(BENEFICIOS;0;40; $[0,075 ; 0,25 ; 0,37 ; 0,42 ; 0,47 ; 0,505 ; 0,520 ; 0,535 ; 0,55 ; 0,56 ; 0,56 / /$ Min:0;Max:0.6//n) & auxiliary \\
\hline & IncrLabor & Fr_Incremento-TRABAJO & auxiliary \\
\hline 8 & Precio & C1*SALARIOS $/ 0,75$ & auxiliary \\
\hline 0 & R_Cap_Trab & 4*PRODUCTIVIDAD & auxiliary \\
\hline & Cambio_Trabajo & (Trabajo_Deseado-TRABAJO)*Frc_Empl & auxiliary \\
\hline & R_Trabajo & (TRABAJO+FuerzaLaboral)/Trabajo_Deseado & auxiliary \\
\hline 0 & Bienes_per_Capita & (INVENTARIO/INIT(INVENTARIO) $)^{* 1}$ & auxiliary \\
\hline
\end{tabular}

\section{REFERENCIAS BIBLIOGRÁFICAS}

Forrester, J. (1961). Principles of systems. Waltham, MA: Pegasus Communications.

Gilbert, N. y Troitzsch, K. (2005). Simulation for the social scientist. Glasgow: McGraw-Hill.

Heilbroner, R. (1998). The worldly philosophers. Nueva York: Simon \& Schuster.

Meadows, D., Meadows, D. L. et al. (1972). The limits to growth. A report to the Club of Rome's Project on the predicament of mankind. Nueva York. Universe Books.

Nijkamp, P. y Reggiani, A. (1992). Interaction, evolution, and chaos in space. Berlín: Springer-Verlag. 
Orlandoni-M., G. (1997). Análisis dinámico de poblaciones biológicas mediante dinámica de sistemas. Revista Economia, 13, 115-146.

Orlandoni M., G. y Ramoni-P., J. (2018a). Ecuaciones diferenciales de la física clásica. Interpretación y solución mediante dinámica de sistemas. Revista UIS Ingenierias, 17(1), 51-58.

Orlandoni M., G. y Ramoni-P., J. (2018b). Análisis y simulación de modelos económicos complejos mediante el enfoque de dinámica de sistemas. Memorias VIII Conferencia Iberoamericana de Complejidad, Informática y Cibernética, 1, 72-77.

Powersim. (2017). Powersim Studio 10 Express.

Radzicki, M. y Sterman, J. (1994). Evolutionary economics and system dynamics. En R. E. England (ed.), Evolutionary concepts in contemporary economics (pp. 39-60). Ann Arbor: University of Michigan Press.

Richardson, G. (1995). Loop polarity, loop dominance and the concept of dominant polarity. System Dynamics Review, 11(1), 67-88.

Richmond, B. (1985). Users guide to Stella. New Hampshire: HPS.

Ruth, M. y Hannon, B. (2012). Modeling dynamic economic systems. Nueva York: Springer.

Smith, A. (1978). An inquiry into the nature and causes of the Wealth of Nations [1776]. Oxford: Oxford University Press. 